\title{
The Hsp40 chaperone Jjj1 is required for the nucleo-cytoplasmic recycling of preribosomal factors in Saccharomyces cerevisiae
}

\author{
EMILIE DEMOINET, ${ }^{1,2}$ ALAIN JACQUIER, ${ }^{2}$ GEORGES LUTFALLA, ${ }^{1}$ and MICHELINE FROMONT-RACINE ${ }^{2}$ \\ ${ }^{1}$ Défenses Antivirales et Antitumorales, cc86 CNRS-UMR5235, Université Montpellier II, 34095 Montpellier Cedex 5, France \\ ${ }^{2}$ Unité de Génétique des Interactions Macromoléculaires, 2171CNRS-URA, Institut Pasteur, F-75724 Paris Cedex 15, France
}

\begin{abstract}
Ribosome biogenesis is a major conserved cellular pathway that requires both ribosomal proteins and many preribosomal factors. Most of the pre-60S factors are recycled into the nucleus; some of them shuttle between the nucleus and the cytoplasm while a few others, like Rei1, are strictly cytoplasmic and are mostly involved in the dissociation/recycling of the pre-60S shuttling factors. Here, we investigated the role of the Jjj1 $\mathrm{Hsp40}$ chaperone in ribosome biogenesis. The absence of Jjj 1 leads to a cold sensitive phenotype, a defect in the relative amount of the large ribosomal subunit with the appearance of halfmers, and to cytoplasmic accumulation of shuttling factors such as Arx1 and Alb1, which stay bound to the pre-60S particles. Jjj1 is, thus, a novel pre-60S factor involved in the last cytoplasmic steps of the large ribosomal subunit biogenesis. We report the biochemical association of Jjj1 and Rei1 to similar pre-60S complexes, their two-hybrid interactions, and their functional links. Altogether, these results indicate that Rei1 and Jjj1 share many common features. However, while the functions of Jjj1 and Rei1 partially overlap, we could distinguish specific role of the two proteins in Arx1/Alb1 and Tif6 recycling. We propose that Jjj1 is preferentially required for the release of Arx1 and Alb1 shuttling factors from the cytoplasmic pre-60S particles while Rei1 is preferentially involved in their recycling.
\end{abstract}

Keywords: ribosome biogenesis; pre-60S maturation; chaperone; nucleocytoplasmic transport; pre-ribosomal factors recycling; yeast Saccharomyces cerevisiae

\section{INTRODUCTION}

The ribosome is one of the most important cellular macromolecular structures in terms of function, size, and the energy that a cell consumes for its biogenesis (Warner 1999). Ribosome biosynthesis begins with the transcription of the $35 \mathrm{~S}$ and $5 \mathrm{~S}$ rRNA precursors by RNA pol I and III, respectively. The processing of the $35 \mathrm{~S}$ rRNA precursor generates the 18S rRNA (backbone of the small ribosomal subunit) and the $5.8 \mathrm{~S}$ and $25 \mathrm{~S}$ rRNAs (backbone of the large ribosomal subunit). The rRNAs are embedded in noncoding spacer regions, the external transcribed sequences,

Reprint requests to: Micheline Fromont-Racine, Génétique des Interactions Macromoléculaires, Institut Pasteur, 25 rue du docteur Roux, 75724 Paris Cedex 15, France; e-mail: mfromont@pasteur.fr; fax: +33 14568790; or Georges Lutfalla, Défenses Antivirales et Antitumorales, cc86 CNRS-UMR5235, Université Montpellier II, Place Eugène Bataillon, 34095 Montpellier Cedex 5, France; e-mail: lutfalla@univ-montp2.fr; fax: +33467 149201.

Article published online ahead of print. Article and publication date are at http://www.rnajournal.org/cgi/doi/10.1261/rna.585007. $5^{\prime}$ - and $3^{\prime}$-ETS, and the internal transcribed sequences, ITS1 and ITS2 (Venema and Tollervey 1999). Association of the 35S rRNA precursor with ribosomal and preribosomal factors generates the large $90 \mathrm{~S}$ preribosomal complex that undergoes various steps of maturation such as chemical modifications (Decatur and Fournier 2002) and exoand endonucleolytic cleavages (Venema and Tollervey 1999) to remove the ETS and ITS regions. Among a wellorchestrated series of cleavage events, the A2 processing generates the large $60 \mathrm{~S}$ precursor particles containing the 27SA2 intermediate rRNA and the small 40S precursor particles containing the 20S intermediate rRNA (Fatica and Tollervey 2002; Fromont-Racine et al. 2003; Tschochner and Hurt 2003). Then, both pre-ribosomal precursor particles follow independent routes; the pre-40S particle is mostly processed in the cytoplasm, whereas the maturation of the pre-60S particle is mostly achieved in the nucleus before export to the cytoplasm where final maturation takes place.

In addition to the large ribosomal protein themselves, around 80 factors have now been predicted or shown to 
participate in the biogenesis of the large ribosomal subunit (Fatica and Tollervey 2002; Fromont-Racine et al. 2003; Tschochner and Hurt 2003). In contrast to the ribosomal proteins, pre-ribosomal factors associate temporarily with the maturing subunits. Most of them associate with and dissociate from the subunits into the nucleus. A few other factors like Arx1, Alb1, Tif6, Rlp24, and Nmd3 are shuttling factors (Senger et al. 2001; Nissan et al. 2002; Saveanu et al. 2003; Hedges et al. 2005; Lebreton et al. 2006b). They bind the pre-60S in the nucleus and dissociate in the cytoplasm. Finally, other factors like Lsg1 (Hedges et al. 2005), Reil (Hung and Johnson 2006; Lebreton et al. 2006b), Efl1 (Senger et al. 2001), and Drg1 (H. Bergler and M. Fromont-Racine, unpubl. data) are strictly cytoplasmic and required for the release and recycling of shuttling factors. These final cytoplasmic steps involve structural rearrangements but no rRNA cleavage. GTPase or ATPase proteins appear to be involved in this process. Indeed, Lsg1 GTPase activity is required for Nmd3 release (Hedges et al. 2005) and in the absence of the Efll GTPase, Tif6 accumulates into the cytoplasm (Senger et al. 2001). The Drg1 ATPase protein (Zakalskiy et al. 2002) is also required for the recycling of shuttling pre-60S factors $(\mathrm{H}$. Bergler and M. Fromont-Racine, unpubl. data). Thus, the ribosomal cleavage and maturation events are tightly coupled to nucleocytoplasmic transport. Recycling requires transport machineries involving karyopherin Kap121, Kap123, and Kap104, which are used for the import of shuttling factors (Rout et al. 1997; Leslie et al. 2004) and exportin (Crm1/Xpo1) for the translocation of the pre-60S particles through the nuclear pore (Ho et al. 2000; Gadal et al. 2001).

In this study, we investigate the role of Jjj1, an Hsp40 chaperone (Meyer et al. 2007) that would be specifically involved in the biogenesis/maturation of the large ribosomal subunit. In eukaryotes, two chaperone networks have been described, a stress inducible network that protects the cellular proteome from stress and another stress repressed chaperone network that is dedicated to protein biogenesis (Albanese et al. 2006). Besides these "general" chaperones, some chaperones are specifically involved in structural transitions for polypeptides in large molecular complexes, as, for example, auxilin/Hsc70 required for clathrin uncoating (Ungewickell et al. 1995; Pishvaee et al. 2000; Fotin et al. 2004). Hsp40s function to specify the cellular targets of Hsp70 chaperones. In the classical view of the function of an Hsp40 chaperone, the protein forms a complex with an unfolded or nonnative protein to prevent its aggregation or misfolding. Hsp70 proteins recognize short hydrophobic polypeptide in this complex, but the polypeptide binding is regulated by the nucleotide bound state. Conformational changes generated upon ATP hydrolysis stabilize Hsp70's interaction with its polypeptide substrate, whereas the exchange of ADP for ATP promotes their releases (Fan et al. 2003; Hennessy et al. 2005).
Apart from Jjj1, no chaperone has been found to be specifically involved in the biogenesis of the large ribosomal subunit. We show here that Jjj1 is involved in the last steps of the biogenesis of the ribosomal large subunit and interacts with Rei1, another late pre-60S factor cytoplasmic involved in the recycling of the shuttling factors (Arx1, Alb1). Even if Jjj1 and Reil share several common characteristics, they are required for different maturation events.

\section{RESULTS}

\section{$\mathrm{Jjj} 1$ is involved in ribosome biogenesis}

We recently demonstrated that Reil is involved in the last cytoplasmic steps of ribosome biogenesis. To gain more insight into potential partners of Reil, a two-hybrid screen was performed. One of the identified candidates was JJJ1/ YNL227W. In Figure 1A, left panel, the inset coding the Jjj1 protein encompassed a region from amino acid 162 to the end of the protein. In addition to the two conserved $\mathrm{C}_{2} \mathrm{H}_{2}$ Zn fingers from amino acids 338 to 362 and from 549 to 573, Jjj1 contains a N-terminal DNA J domain (up to amino acid 67) that assigns Jjj1 to the Hsp40 chaperone family. The DNA J domain was excluded from the selected Reil interacting domain. The physical interaction between Jjj1 and Rei1 was confirmed by a two-hybrid matrix approach. To further investigate additional interactions, we tested physical interactions between Jjj1 and several pre-60S factors previously described as partners of Rei1, such as Arx1, Alb1, and the ribosomal protein Rpl24. A two-hybrid interaction was observed between Jjj1 and Reil (Fig. 1A, right panel). No other physical interaction was found with Jjj1 whereas Reil interacted with Rpl24, and Arx1 interacted with itself and with Alb1 (Lebreton et al. 2006b). We noted that Alb1 was an autoactivating bait. The strongest two-hybrid signals were obtained between Arxl and itself and between Arx1 and Alb1, as demonstrated by the dark blue color obtained by X-Gal overlay assay.

JJJ1 is not essential in yeast; however, its deletion leads to a cold sensitive phenotype. Apart from the DNA J motif, suggesting a chaperone activity, the function of this protein was unknown. However, in addition to the physical twohybrid links between Reil and Jjj1, several lines of evidence suggested that Jjj1 could be involved in ribosome biogenesis. For example, the JJJ1 mRNA level fluctuates similarly to the levels of mRNAs coding for preribosomal factors in response to various environmental stresses (Gasch et al. 2000). Moreover, Jjj1 has been identified in several preribosomal complexes using the TAP purification approach (Gavin et al. 2006).

To investigate the involvement of Jjj1 in ribosome biogenesis, we looked at the polysome profile of a jjj1s cellular extract from the ED10 (jjj1 $\Delta$ ) strain after ultracentrifugation in a sucrose gradient. The relative amount of 
A

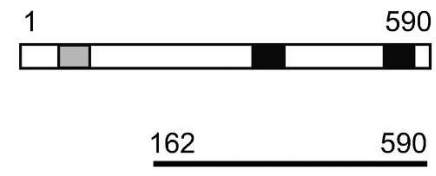

$\square$ J Domain

$\mathrm{C}_{2} \mathrm{H}_{2}$ motif

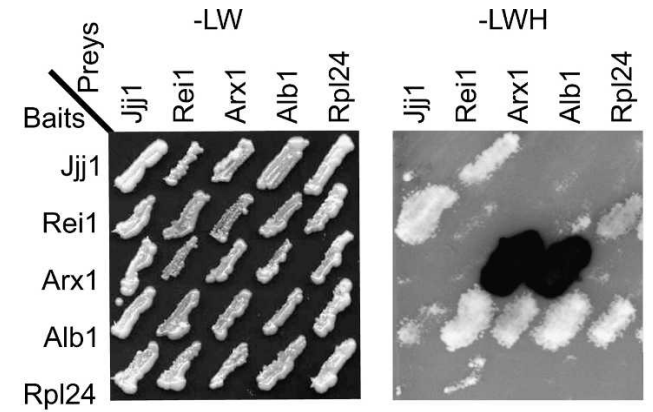

C

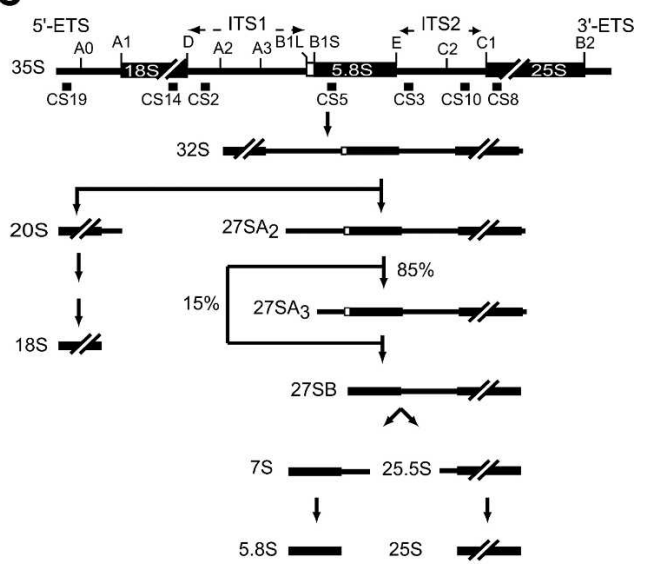

D



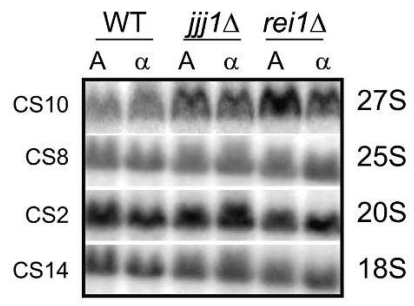

FIGURE 1. Jjj1 is involved in the 60S subunit biogenesis. (A) Two-hybrid screen identifies a physical link between Jjj1 and Rei1. (Right panel) Jjj1 was selected as a partner of Reil in a two-hybrid genomic screen. The domain of Jjj1 interacting with Reil corresponds to amino acids 162-590. The two putative $\mathrm{C}_{2} \mathrm{H}_{2} \mathrm{Zn}$-fingers motifs and the J domain are depicted by black and gray boxes, respectively. (Left panel) Various entire ORF were cloned into pAS2 $\Delta \Delta$ as bait and into pACTIIst as prey vectors, respectively. The bait and the prey vectors were transformed into the CG1945 and Y187 strains, respectively. Both strains were mated and the diploids were selected on minimal medium plates without leucine and tryptophan $(-\mathrm{LW})$. The diploids that display a two-hybrid positive interaction were selected on minimal medium plates without leucine, tryptophan and histidine $(-\mathrm{LWH})$. An X-Gal overlay was performed on the $-\mathrm{LWH}$ plate. (B) In the absence of Jjj1, the relative amount of $60 \mathrm{~S}$ subunit is affected. A wild-type (BY4741) or a jjj1s strain (ED10) was grown in YPGlu and shifted at $23^{\circ} \mathrm{C}$ for $2 \mathrm{~h}$. The whole-cell extracts from these strains were separated by ultracentrifugation on a $10 \%-50 \%$ sucrose gradient. Peaks corresponding to the various subunits and polysomes are indicated. Asterisks note halfmers. $(C)$ Schematic representation of the ribosomal RNAs maturation. The relative position of the oligonucleotides used to detect specific rRNA by primer extension is indicated. $(D)$ In the absence of Jjj1, the 27SB processing is very weakly affected. Total RNAs were extracted from culture cells of wild-type strain (BY4741), jij1 $\Delta$ (ED10), and rei1s mutant strains (ED70) in YPGlu medium after a shift to $23^{\circ} \mathrm{C}$ for $2 \mathrm{~h}$. Mature and intermediate rRNA were detected either by primer extension (left panel) or by Northern blot on agarose gel (middle panel) or on acrylamide gel (right panel). The oligonucleotides used are noted. The U2 snRNA was used as a loading control.

60 Subunit was clearly affected in the absence of $\mathrm{Jjj} 1$ (Fig. 1B, right panel) as compared to the wild-type strain (Fig. 1B, left panel). Moreover, we observed a decrease of the total amount of polysomes and the presence of half- mers, which reflects the relative deficit in $60 \mathrm{~S}$ as compared to $40 \mathrm{~S}$ during translation initiation. This phenotype was obvious at $30^{\circ} \mathrm{C}$ (data not shown) but was even more pronounced after a shift to $23^{\circ} \mathrm{C}$ for $2 \mathrm{~h}$ (Fig. 1B). To define 
which step of ribosome biogenesis is affected by the absence of $\mathrm{Jjj} 1$, we tested the relative amounts of the different mature and intermediate rRNA in jjj1s strains (ED10 mat $\alpha$, ED11 mat a) in comparison with a wild-type (BY4742, BY4741) or a rei1s (ED70 mat $\alpha$, ED71 mat a) strain (Fig. 1D). Steady-state rRNA levels were analyzed by primer extension (Fig. 1D, left panel) and by Northern blotting either on agarose (Fig. 1D, middle panel) or acrylamide gels (Fig. 1D, right panel) from wild-type, Jjj1, and Rei1depleted cells after a shift to $23^{\circ} \mathrm{C}$ for $2 \mathrm{~h}$. With the exception of the $27 \mathrm{SB} / \mathrm{A} 2$ pre-rRNA ratio, which very slightly increased (2.9 for the $j j j 1 \Delta$ strains compared to 1.5 for the WT strains and 3.2 to reils strains based on two experiments, one on each mating type), none of the other ratios were significantly affected. Similar results were obtained after a 15 -min shift at $23^{\circ} \mathrm{C}$ with rei1s, jjj1s, and WT strains (mat a, mat $\alpha$ ) (data not shown). These results are in agreement with the decrease of the relative amount of the $60 \mathrm{~S}$ subunit in the absence of Jjj1 but suggest that Jjj1 has no direct influence on nuclear pre-rRNA processing.

Since ribosome maturation mutations are usually correlated with a ribosome export defect, we tested the localization of the ribosomal Rpl25-GFP fusion protein in the jjj1 $1 \Delta$ strain. We observed that, like in a reils strain or in a wild-type strain, the fluorescence was mainly located in the cytoplasm (data not shown), suggesting that the export of Rpl25-GFP was not affected. In addition, like Rei1, Jjj1 is a cytoplasmic protein (Huh et al. 2003).

Altogether, these results show that Jjj1 is a cytoplasmic factor affecting nuclear ribosomal large subunit maturation. This is reminiscent of the effect described for reils, which participates in the recycling of shuttling pre-60S factors (Hung and Johnson 2006; Lebreton et al. 2006b).

\section{Jjj1 physically interacts with the pre-60S particles}

The physical two-hybrid interaction between Jjj1 and Reil correlates with similar defects of the $60 \mathrm{~S}$ biogenesis in the absence of either of these proteins. To check whether Jjj1, like Reil, is biochemically associated with the 60S particles, we performed a sucrose gradient experiment using a chromosomal Jjj1-TAP fusion protein (OT52) (Open Biosystem). After sedimentation of the whole cellular extracts on sucrose gradient, we observed that Jjj1-TAP fusion protein was present in the fractions corresponding to the $60 \mathrm{~S}$ ribosomal particle but not in the polysome fractions (Fig. 2A) suggesting that Jjj1, like
Rei1, is a pre-60S-associated factor. Since Jjj1 and Rei1 interact in a two-hybrid assay and since they are both associated to pre-60S ribosomal particles, we attempted to determine if both proteins were present in the same particles. We purified the complex associated with Jjj1TAP using a strain producing a Reil-13myc fusion protein and also performed the reverse experiment. Jjj1-13myc was only present in the complexes associated with Reil but not with Rlp24 (Fig. 2B) and Rei1-13myc was copurified with Jjj1-associated complexes but not with Rlp24. As a control, we observed that Rlp24 was copurified neither with Jjj1TAP nor with Rei1-TAP while it copurified with Mak11TAP. These results suggest that (1) Jjj1 and Reil are present in the same complexes and (2) like Rei1, Jjj1 binds to the particle after the release of Rlp24.

We checked for the dependence of the presence of Jjj1 for the binding of Reil to the particle and inversely by using strains depleted for the first one and producing a TAP fusion of the second one. The association of the proteins to the pre-60S complexes was tested by Western blotting on fractions of a sucrose gradient. Surprisingly, neither Jjj1 nor Reil was required for the recruitment of the other protein to the pre-60S particle (data not shown).

\section{Jjj1 and Rei1 are functionally linked}

Several lines of evidence indicate that there is a strong similarity between Jjj1 and Reil: (1) Both proteins are
A

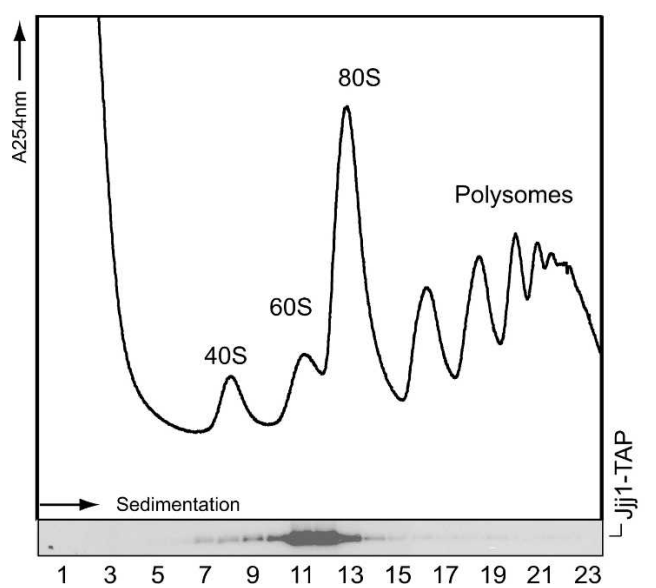

B

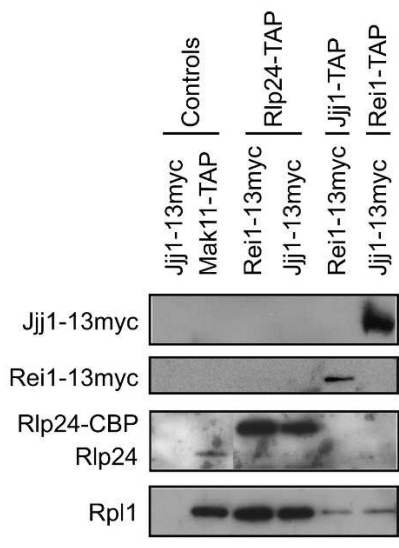

FIGURE 2. JjjI and Reil are involved in the same complexes. (A) $\mathrm{Jjj} 1$ is associated with the $60 \mathrm{~S}$ particles. The whole-cell extract of a strain expressing a Jjj1-TAP fusion protein (OT52) was separated by ultracentrifugation on a $10 \%-50 \%$ sucrose gradient. Fractions of $0.5 \mathrm{~mL}$ were collected. Proteins were precipitated by TCA and separated on a SDS $-4 \%-12 \%$ polyacrylamide gel. The presence of Jjj1-TAP fusion protein was revealed by Western blotting using PAP antibodies (Sigma). (B) Jjj1 and Reil are present in the same pre-60S complexes. TAP purifications were performed on strains expressing Rei1-TAP, Jjj1-13myc (ED46); Jjj1-TAP, Rei113myc (ED19); Rlp24-TAP, Jjj1-13myc (ED87); and Rlp24-TAP, Rei1-13myc (LMA352) fusion proteins. Mak11-TAP (LMA375) and Jjj1-13myc (ED82) strains were used as control. The presence of Jjj1-13myc, Rei1-13myc, Rlp24, and Rpl1 was tested using antibodies against myc, Rlp24, and Rpl1, respectively. 
involved in the pre-60S biogenesis, (2) they are located in the cytoplasm, (3) they physically interact, (4) both proteins have $\mathrm{Zn}$ finger motifs, and (5) deletion of either JJJ1 or REI1 leads to a cold sensitive phenotype. To determine if these proteins would have redundant functions, we tested the effect of the double deletion (Fig. 3A). In comparison to a generation time of $2 \mathrm{~h} 41 \mathrm{~min}$ for a wild-type strain, we observe a generation time of $5 \mathrm{~h} 20 \mathrm{~min}$ for jjj $1 \Delta$ rei1s double mutant strain (ED21) similar to the generation time of $5 \mathrm{~h}$ $37 \mathrm{~min}$ of the reils strain (LMA523), whereas the generation time for $j j j 1 \Delta$ is of $4 \mathrm{~h} 5 \mathrm{~min}$, suggesting that both JJJ1 and REI1 genes are epistatic.

To further investigate the role of Jjj1, we performed a high-copy suppressor genetic screen with the JJJ1-deleted strain (ED10) at $20^{\circ} \mathrm{C}$. Interestingly, in addition to JJJ1 containing plasmids, one family of the rescued plasmids containing the REI1 gene was able to partially complements the cold sensitivity of the jjj1 $1 \Delta$ mutant strain. Figure 3B (left panel) shows the complementation using a $2 \mu \mathrm{m}$ plasmid derived from pFL44L containing JJJ1. As expected, overexpression of REI1 was also able to partially restore the cold-sensitive phenotype of jjj1L (Fig. 3B, left panel). We tested the reverse situation by overexpressing JJJ1 into a reils mutant strain (ED72). While pFL44L-REI1 complements the absence of Reil, the overexpression of JJJ1 had no effect on the reils mutant (same as empty vector; data not shown). Overexpression of either JJJ1 or REI1 was tested on the jji1s rei1s double mutant strain (ED21) (Fig. 3B, right panel). While the overexpression of JJJ1 had no effect, we observed a partial rescue of the growth of jij1 $1 \Delta$ reil $\Delta$ double mutant strain by overexpression of REI1.

Polysome profiles of the jijis strains transformed with different vectors were analyzed after a shift of temperature for $2 \mathrm{~h}$ at $23^{\circ} \mathrm{C}$ from $30^{\circ} \mathrm{C}$. The amount of $60 \mathrm{~S}$ subunit was clearly affected in the absence of $\mathrm{Jjj} 1$ as compared to the jjj1 $1 \Delta$ strain complemented by the overexpression of $J J J 1$. Interestingly, REI1 overexpression partially restored the relative amount of $60 \mathrm{~S}$ subunit (Fig. 3C), in agreement with the partial growth rescue.

We conclude that overexpression of REI1 partially complements the absence of Jjj1 but the reverse is not true.
Therefore, while Jjj1 and Reil proteins display some similarities and are involved in the same pathway, their functions are not identical.

The absence of either Arx1 or Alb1 is able to complement the growth defect of the reils strain (Lebreton et al. 2006b; Meyer et al. 2007). To further investigate the similarities and the differences between Jjj1 and Rei1, we checked whether the presence of Arx1 or Alb1 was responsible for the cold sensitivity of the $j j j 1 \Delta$ strain. In fact the $j j j 1 \Delta$ arx $1 \Delta$ and jjj1 $1 \Delta$ alb1 $\Delta$ double mutant strains grew as well as arx $1 \Delta$ or albls strains at $23^{\circ} \mathrm{C}$ (Fig. 4A), showing that the absence of Arx1 (or Alb1) rescued the cold sensitivity of jjj1 $1 \Delta$. Moreover, using polysome profiles, we observed that the relative amount of $60 \mathrm{~S}$ was restored to the 
A

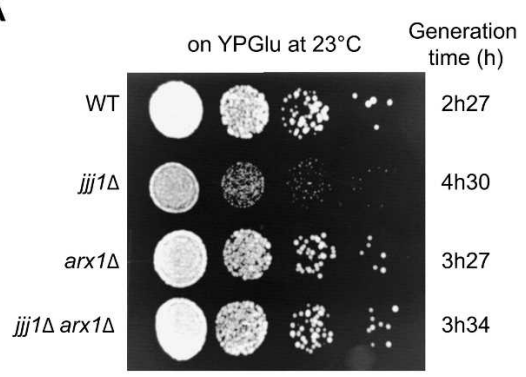

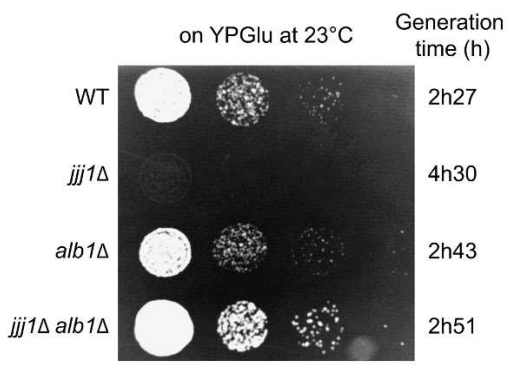

B
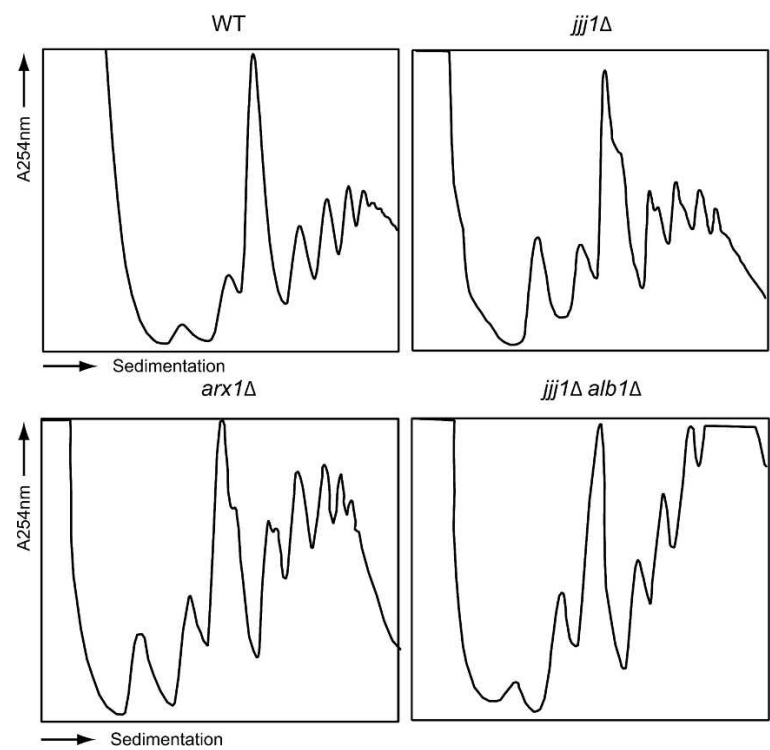

FIGURE 4. In the absence of Arx1/Alb1, deletion of JJJ1 is not toxic. (A) Deletion of either $A R X 1$ or $A L B 1$ rescues the cold sensitivity effect of $j i j 1 \Delta$ strain. Ten times serial dilutions of

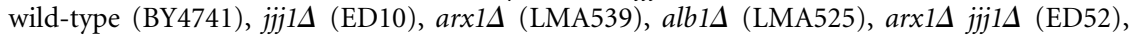
and alb1s jij1 $\Delta$ (ED65) cultures were spotted on solid rich medium. Their growth on YPGlu at $23^{\circ} \mathrm{C}$ was compared. $(B)$ Deletion of either $A R X 1$ or $A L B 1$ restores a wild-type polysome profile. The whole-cell extracts from the strains used in $A$ were separated on a sucrose gradient as described in Figure 1B.

wild-type level in the $j j j 1 \Delta$ arxis and $0 j j 1 \Delta$ alb1s double mutant strains when compared to jjj1s alone (Fig. 4B). This observation is consistent with the recent hypothesis that the accumulation of Arx1 and Alb1 as a small cytoplasmic complex (Lebreton et al. 2006b) could be responsible for the slow growth phenotype of the reils strain.

\section{Jjj1 and Rei1 have distinct functions in the Arx1/Alb1 recycling}

Since the cold sensitivity of the two deleted jjj1s or reils strains was abolished by the deletion of $A R X 1$ or $A L B 1$, we hypothesized that Jjj1 could participate with Rei1 in the recycling of shuttling pre- $60 \mathrm{~S}$ factors. We predicted that the proteins affected in their recycling in the absence of Rei1 would also be affected by the absence of Jjj1. We observed that Arx1-GFP and Alb1-GFP accumulated in the cytoplasm in the absence of Jjj1 as well as in the absence of Rei1 (Fig. 5A, left and middle panels). To check if Arx1 and Alb1 are blocked on the cytoplasmic pre-60S particles or present in small cytoplasmic complexes in the absence of Jjj1, whole-cell extracts from strains expressing Alb1-GFP in the absence of Jjj1 were separated by sedimentation on a sucrose gradient. In the absence of Jjj1, endogenous Arxl and Alb1-GFP fusion protein sedimented in the 60S peak whereas in the absence of Reil, both Arx1 and Alb1-GFP accumulated as small complexes (Fig. 5B) as previously described (Lebreton et al. 2006b). Similar results were obtained in a strain expressing an Arxl-GFP fusion protein (data not shown). These results are in agreement with the genetic links described above. Even if Jjj1 and Reil are involved in the same pathway, their functions are not fully equivalent. Previous results have suggested that the recycling of these factors involved the Kap121 karyopherin pathway. This hypothesis was supported by the fact that overexpression of KAP121 was able to recycle Arx1 and Alb1 into the nucleus and partially restore the slow growth phenotype of the reils strain (Fig. 6A, left panel; Lebreton et al. 2006b). If in jjj1s, Arxl and Alb1 are not released from the particle, overexpression of KAP121 should not result in relocalization of Arx1/Alb1 in the nucleus. Indeed, in contrast to reils, KAP121 overexpression (Fig. 6A, middle panel) had no effect on the jjj1 $1 \Delta$ strain. It was able to restore neither the growth phenotype nor the polysome profile (data not shown) nor the normal localization of Arx1-GFP (Fig. 6B). As a control, we observed that, in a reils strain, KAP121 overexpression allowed Arx1/Alb1 recycling (Fig. 6B). Interestingly, KAP121 overexpression led to a partial rescue of the slow growth phenotype in the jji1 $1 \Delta$ reils double mutant strain as in reils strain (Fig. 6A, cf. right and left panels).

These results confirm that in the absence of Jjj1, Arx 1 and Alb1 are mostly blocked on pre-60S cytoplasmic particles, where they are probably not available to the import machinery, whereas in the absence of Rei1, Arxl and Alb1 accumulate as small cytoplasmic complexes, where they still can be caught by an overwhelming amount of Kap121 karyopherin. 

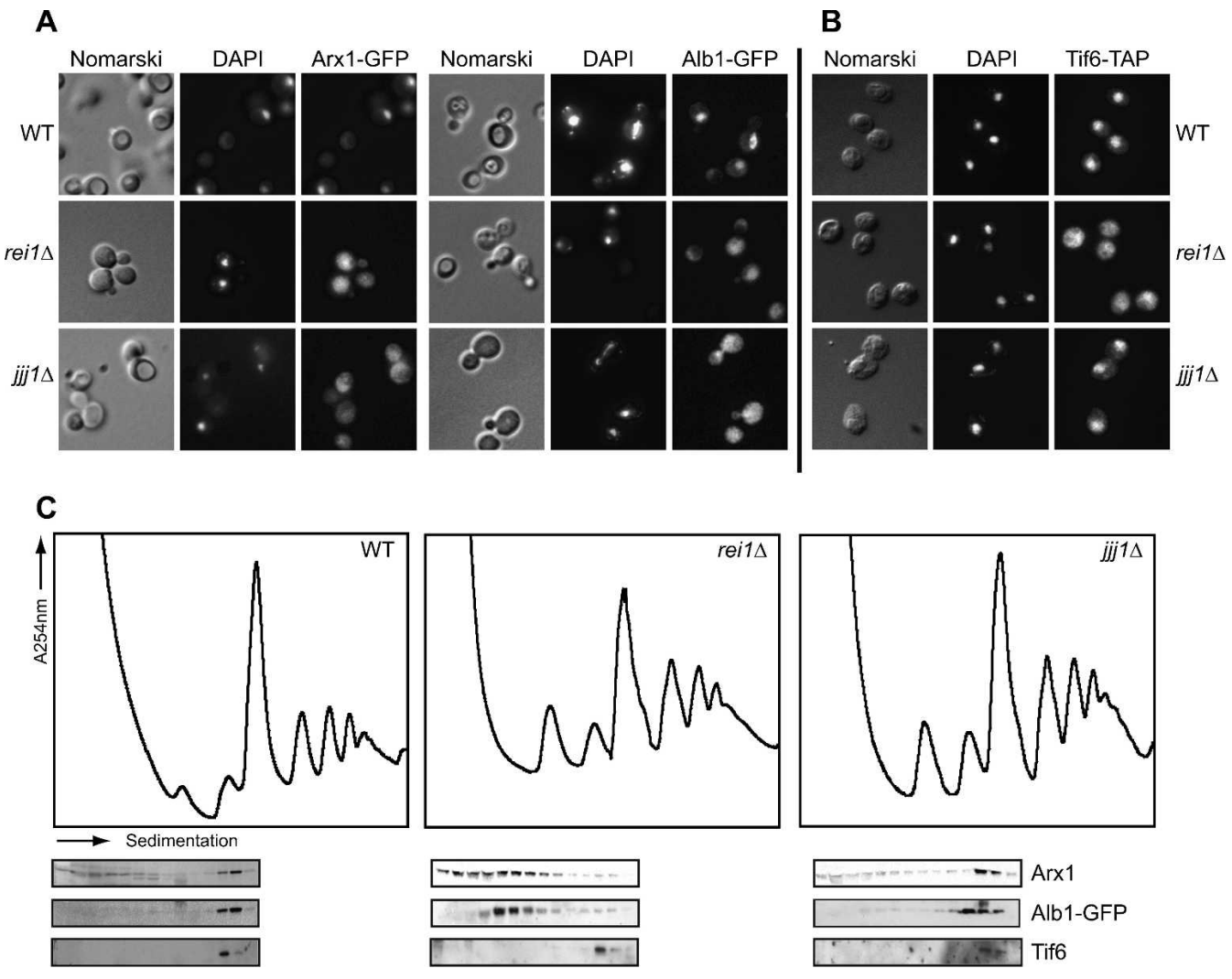

FIGURE 5. In the absence of Jjj1, Arx1/Alb1 are blocked on cytoplasmic 60S particles. (A) Arx1 and Alb1 but not Tif6 (B) accumulate into the cytoplasm in the absence of Jjj1. Arx1-GFP (LMA401), Alb1-GFP (LMA545-B), Arx1-GFP, jjj1 (ED49), Arx1-GFP, rei1s (LMA411), Alb1-GFP,

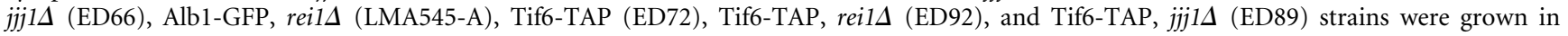
minimal media at $30^{\circ} \mathrm{C}$ and shifted to $23^{\circ} \mathrm{C}$ for $2 \mathrm{~h}$. The cells were observed by fluorescence microscopy. Tif6-TAP localization was observed on fixed cells using PAP antibodies (Sigma) at 1/5000 dilution followed by Cy3-conjugated secondary antibodies (Jackson Immunoresearch) at $1 / 250$ dilution. $(C)$ Arxl and Alb1 are blocked on the 60S particle in the absence of Jjj1. The whole-cell extracts from the strains used in $A$ were separated on a sucrose gradient and the fractions were analyzed as described in Figure 2A. The Western blots were performed using the fractions of the polysome profiles from 2 to 15. The presence Arx1, Tif6, and Alb1-GFP fusion proteins were checked using anti-Arx1, anti-Tif6, and anti-GFP antibodies (Santa Cruz Biotechnology) at 1/700, 1/5000 and 1/700 dilutions, respectively.

\section{The absence of Jjj1 or Rei1 differently affects Tif6 recycling}

We recently proposed that, in a reils strain, the cytoplasmic accumulation of small Arx1-associated complexes could be responsible for blocking Tif6 on the pre-60S particle (Lebreton et al. 2006b). In the absence of Jjj1, no such small cytoplasmic Arx1-associated complexes were formed; we therefore expected Tif6 to be released from the pre-60S particle and correctly recycled. Interestingly, while Tif6 accumulated in the cytoplasm in the absence of Reil, it was localized in the nucleus in the absence of Jjj1 (Fig. 5A, right panel). We also checked the sedimentation of Tif6TAP in the absence of Jjj1; it sedimented in fractions corresponding to the $60 \mathrm{~S}$ peak (Fig. $5 \mathrm{~B}$ ). This suggests that in the absence of Jjj1, Tif6 is correctly released from the pre-60S cytoplasmic particles and recycles to the nucleus.

In conclusion, Tif6 does not seem to be affected by the deletion of JJJ1 while it requires REI1 for dissociation from the cytoplasmic pre-60S particle.

\section{DISCUSSION}

The first precursor particle involved in ribosome biogenesis, the 90S, generates the pre-40S and pre-60S intermediate ribosomal particles after the A2 cleavage. The large ribosomal subunit maturation requires about 80 pre-ribosomal factors. This pre-60S particle goes through successive maturation steps, including rRNA processing, conformational changes, and transport events through the nucleolus, the nucleoplasm, and the nucleopore complex. Thus, it is not surprising that most of these 80 or so pre-60S factors are nuclear factors whereas few are strictly cytoplasmic. When the pre-60S particles reach the cytoplasm, the mature ribosomal RNAs are formed, and many pre-ribosomal factors have already left the particles. Only a few shuttling factors, such as Arx1, Alb1, Tif6, Rlp24, and Nmd3, are still present. Then, some strictly cytoplasmic pre-60S factors act on the pre-60S particles to finalize their maturation.

We report here the role of a strictly cytoplasmic pre-60S factor, Jjj1, in the biogenesis of the large ribosomal subunit. 


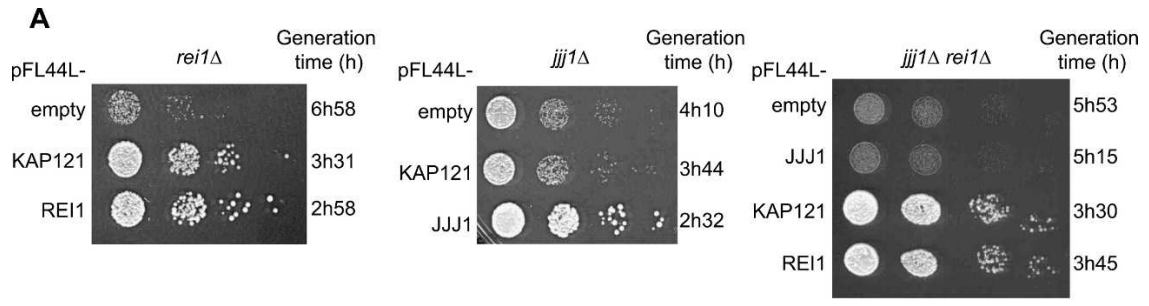

B
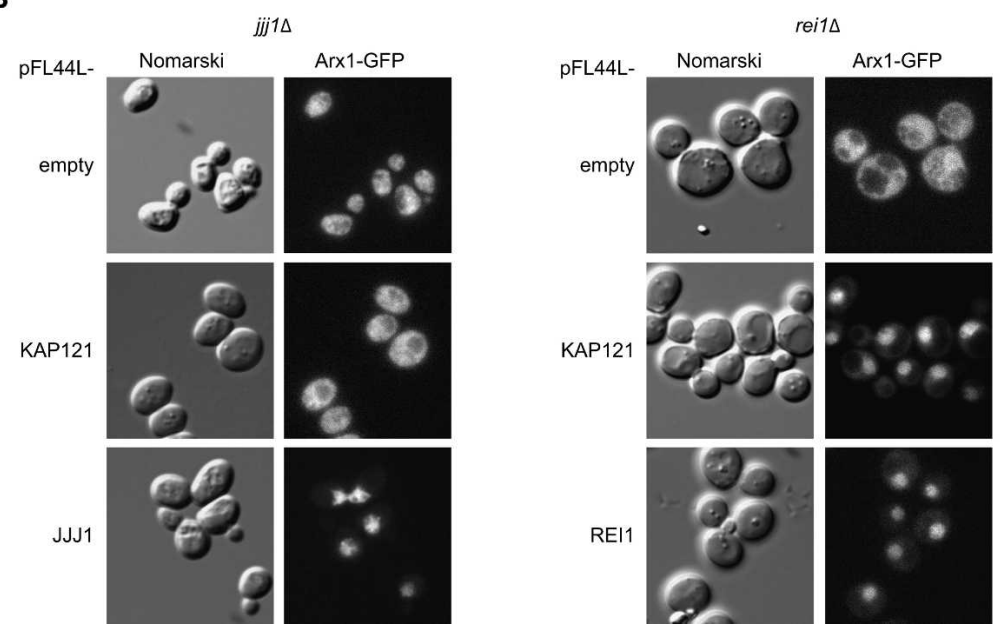

FIGURE 6. KAP121 overexpression has distinct effects on rei1s and $i j j 1 \Delta$ strains. (A) KAP121 overexpression does not restore the growth of jjj1 $1 \Delta$. Ten times serial dilutions of reis (LMA523), jji1s (ED10), and rei1s jji1s (ED21) transformed with either the empty vector pFL44L or pFL44L-REI1 or pFL44L-JJJ1 or pFL44L-KAP121 were spotted on solid minimal medium without uracil at $23^{\circ} \mathrm{C}$ for $2 \mathrm{~d}$. Generations times are indicated. (B) KAP121 overexpression does not restore nuclear localization in the absence of Jjj1. Arxl-GFP, jjj1 $\Delta$ (ED49) and Arx1-GFP, reils (LMA411) strains were transformed with either the empty vector pFL44L or pFL44L-REI1 or pFL44L-JJJ1 or pFL44L-KAP121. The cells were grown in minimal media at $30^{\circ} \mathrm{C}$ and shifted at $23^{\circ} \mathrm{C}$ for $2 \mathrm{~h}$. Arxl-GFP localization was observed by fluorescence microscopy.

While this manuscript was being prepared, Meyer et al. (2007) reported a functional analysis of Jjj1. In agreement with our data, Meyer et al. report that Jjj1 is involved in the biogenesis of the 60S large ribosomal subunit and shares similar features with the Reil pre-60S factor. Additional experiments presented here revealed that, while the functions of Jjj1 and Reil proteins show similarities, they are nevertheless clearly distinct.

\section{While Rei1 and Jjj1 share similar features, they have distinct functions}

In addition to the fact that $\mathrm{Jjj} 1$ and Reil have zinc-finger motifs, both proteins share several similarities. They are late strictly cytoplasmic pre-60S associated factors. Their absence leads to a cold sensitivity and a slow growth phenotype correlated with a relative decrease of the amount of $60 \mathrm{~S}$ subunit and a weak defect in rRNA maturation (Figs. 1, 3; Hung and Johnson 2006; Lebreton et al. 2006b; Meyer et al. 2007). Both proteins interact together not only in a two-hybrid assay (Fig. 1) but also in co-immunopre- cipitation experiments (Fig. 2), revealing that both proteins are biochemically associated to similar pre-60S complexes. Since both Jjj1 and Reil are physically associated to the same cytoplasmic late particles, we tried to define the order of assembly of these two factors to the pre$60 S$ particles. Surprisingly, sucrose gradient analysis of strains deleted for REI1 or JJJ1 revealed that the binding of each one of these proteins to the pre-60S particles is independent from the presence of the other (data not shown). Here, we observed that, as for the recruitment of Reil onto the particle, the binding of Jjj1 is one of the last steps in the ribosome biogenesis. Jjj1, like Reil, binds to the particle after the release of Rlp24 pre-60S factor from the particles. Thus, the transient presence of Jjj1 on pre-60S particles correlates very well with the chaperone activity of Jjj1.

Besides the biochemical features of Jjj1 and Rei1, several data indicate that the roles of these proteins are intertwined. Both Jjj1 and Reil are involved in the recycling of the pre-60S shuttling factors Arx1 and Alb1 (Fig. 5A). The slow growth phenotype of $j j j 1 \Delta$ or reils strains can be rescued by the deletion of $A R X 1$ or $A L B 1$ (Fig. 4; Hung and Johnson 2006; Lebreton et al. 2006b), suggesting that the fate of Arxl and Alb1 is responsible for the cold sensitivity of $j j j 1 \Delta$ and reils strains. As reported by Meyer et al. (2007), we observe that Arx1 and Alb1 also accumulate in the cytoplasm in the absence of Jjil.

However, while Jjj1 and Reil proteins have strong similarities, we describe here key differences revealing distinct functions of these proteins in 60S formation. Our previous model proposed that, in the absence of Rei1, it is the cytoplasmic accumulation of the small complexes including Arx1 and Alb1 that prevents the release of Tif6 from the pre-60S particle. We show here that, in the absence of Jjj1, Arx1 and Alb1 do not accumulate in small cytoplasmic complexes but remain associated with the pre$60 \mathrm{~S}$ particle and that Tif6 is correctly recycled to the nucleus (Fig. 5).

Three karyopherins, Kap121/Pse1, Kap123, and Kap104 (Rout et al. 1997; Leslie et al. 2004) are involved in the import of ribosomal components into the nucleus. In a rei1s strain, overexpression of KAP121 partially restores the growth defect and allows the recycling of Arx1/Alb1 and Tif6 into the nucleus (Lebreton et al. 2006b) while in the 
absence of Jjj1, overexpression of KAP121 has no effect on Arx1 and Alb1 recycling (Fig. 6). This is correlated with the fact that Arx1 and Alb1 are not released from pre-60S particles in this context.

We conclude that Jjj1 would be preferentially involved in the dissociation of Arx1 and Alb1 from the pre-60S particle, whereas Reil would rather be preferentially involved in the recycling of these factors by the karyopherins pathway.

\section{Why is the presence of Arx 1 toxic when $J J 1$ or REI1 are deleted?}

Arx1 deficient cells have almost no growth phenotype. Surprisingly, the absence of Arx1 leads to halfmers formation, whereas the amount of $60 \mathrm{~S}$ does not seem strongly affected (Fig. 4) suggesting that this factor is not directly required for the production of the pre-60S subunit but could rather be important for the quality of the produced $60 \mathrm{~S}$ subunit. The data presented here and by Hung and Johnson (2006), Lebreton et al. (2006b), and Meyer et al. (2007) suggest that a failure in its recycling may be more deleterious to the cells than its absence. Indeed, we have described two mutants (rei1s and jjj1 $1 \Delta$ ) whose deleterious phenotypes are correlated with the cytoplasmic accumulation of Arx1 and Alb1 and are rescued by deletion of the $A R X 1$ or $A L B 1$ gene. Therefore, it appears that the cytoplasmic accumulation of Arx1 is mainly responsible for the growth defects observed in the absence of either Jjj1 or Rei1. But we now show that the deleterious effect occurs both when Arx1 and Alb1 are released from the pre-60S (rei1s) or stalled on the pre-60S particle (jjj1s). The recycling of Tif6 is also affected in the absence of Reil as Arx1 and Alb1 accumulate in small cytoplasmic complexes.

Surprisingly, nuclear Arxl recycling is not necessarily correlated with a rescue of a normal growth of the cells. Indeed, in the absence of Rei1, JJJ1 (data not shown) and KAP121 overexpressions allow the recycling of Arx1 into the nucleus (Lebreton et al. 2006b). However, only KAP121 overexpression is able to partially rescue the wild-type phenotype. In all cases, Arx1 is not anymore associated to the pre-60S particle and the polysome profile is not rescued. When the correct Arxl process is interrupted, whatever the gene overexpressions tested to rescue the wild-type phenotype, only partial function of Arxl is recovered, suggesting that the moment and the conditions in which Arx1 and Alb1 are released from the particles and reimported are crucial. Jjj1, as a Hsp40 chaperone catalyzing structural transitions, could play a key role in allowing the commitment of the ribosomal large subunit toward translation initiation.

\section{The role of Jjj 1 as a chaperone}

The role of Hsp40, characterized by their J domain, is to stimulate the ATPase activity of their Hsp70 partner.
Recently, Meyer et al. (2007) have shown that the Hsp70 cochaperone of Jjj1 could be Ssa1. In eukaryotic cells, there are two major cytosolic classes of Hsp70: the SSA family composed of fourgenes, $S S A 1-4$, and the $S S B$ family, encoded by SSB1 and SSB2. Whereas Ssb chaperones are ribosome associated and bind nascent polypeptides to prevent their misfolding at the ribosome exit channel, Ssa chaperones have pleiotropic functions: translocation of the newly synthetized polypeptides to the reticulum endoplasmic or mitochondria and other folding process.

Apart from Jjj1, no chaperones have been found to be specifically involved in the biogenesis of the ribosomal large subunit. Indeed, the RAC (ribosome associated complex), composed of the Hsp40, Zuo1, and its two Hsp70 partners, Ssz1 and Ssb1/2, binds the NAC (nascent chain associated complex), composed of three factors, Egd2, Egd1 and Btt1, to prevent misfolding of the newly synthesized polypeptide at the exit ribosome channel; it is not involved in the maturation of the ribosomal subunits. The specific role of Jjj1 in ribosome biogenesis could be compared to the specific function of the auxilin Hsp40. Indeed, Swa2/auxilin Hsp40 is specifically involved in clathrin uncoating by inducing transconformational changes of clathrin (Ungewickell et al. 1995; Fan et al. 2003; Fotin et al. 2004; Hennessy et al. 2005). Jjj1 seems to be specifically involved in Arx1 release from the particle. Indeed, when the very conserved histidine-proline-aspartic acid (HPD) motif of the J domain is mutated, Arx1 cannot be recycled and accumulates in the cytoplasm (Meyer et al. 2007). This suggests that the function of Jjj1 relies on its chaperone activity.

\section{CONCLUSION}

When the pre-60S particles arrive in the cytoplasm, they are not yet competent for translation and a few final steps are required. A strong cytoplasmic control is important because commitment of pre-60S particles into translation initiation should be extremely deleterious for the cells. A coordinated pre-60S particles export and pre-60S factors import should be an important level of regulation to control the entry in translation.

\section{MATERIALS AND METHODS}

\section{Plasmids, oligonucleotides, strains, and growth conditions}

The strains used in this study are listed in Table 1. Chromosomal insertions were obtained by homologous recombination using PCR fragments in MGD13-353D or BY strains (Baudin et al. 1993). Standard yeast genetic methods and selective growth media were used. The plasmids encoded JJJ1 as two-hybrid bait (pED1) or prey (pED4) were obtained by Gateway cloning JJJ1 in pAS2 $\Delta \Delta$ and pACTIIst destination vectors. The other two-hybrid plasmids 
TABLE 1. Yeast strains used in this study

\begin{tabular}{|c|c|c|}
\hline Strain & Genotype & Reference \\
\hline MGD353-13D & $\begin{array}{l}\text { MATa, trp1-289, ura3-52, ade2, } \\
\quad \text { leu2,-3-112, arg4 }\end{array}$ & Rigaut et al. (1999) \\
\hline BY4741 & MATa, ura $3 \Delta 0$, his $3 \Delta 1$, leu $2 \Delta 0, \operatorname{met} 15 \Delta O$ & Brachmann et al. (1998) \\
\hline BY4742 & $M A T \alpha$, ura $3 \Delta O$, his $3 \Delta 1$, leu $2 \Delta O$, lys $2 \Delta O$ & Brachmann et al. (1998) \\
\hline ED10 & $\begin{array}{l}M A T \alpha, \text { ura } 3 \Delta 0, \text { his } 3 \Delta 1, \text { leu } 2 \Delta 0, \\
\operatorname{met} 15 \Delta 0, \text { jjj } 1 \Delta:: \text { KanMX4 }\end{array}$ & Winzeler et al. (1999) \\
\hline ED11 & 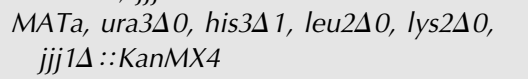 & Winzeler et al. (1999) \\
\hline ED70 & 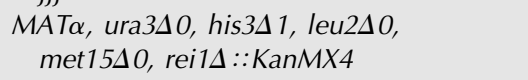 & Winzeler et al. (1999) \\
\hline ED71 & $\begin{array}{l}\text { MAT a, ura } 3 \Delta 0, \text { his } 3 \Delta 1 \text {, leu } 2 \Delta 0, \text { lys } 2 \Delta 0, \\
\quad \text { rei1 } 1 \Delta: \text { KanMX4 }\end{array}$ & Winzeler et al. (1999) \\
\hline ED21 & 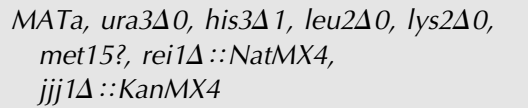 & This study \\
\hline ED52 & 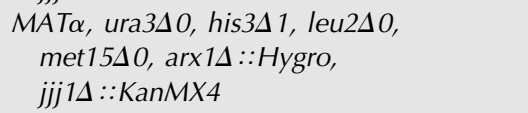 & This study \\
\hline ED65 & 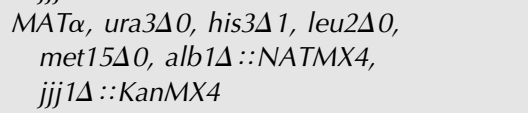 & This study \\
\hline LMA523 & 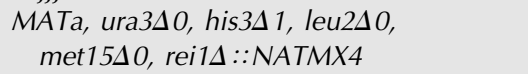 & Lebreton et al. (2006b) \\
\hline LMA539 & 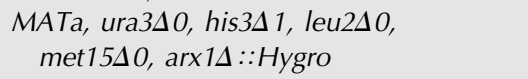 & Lebreton et al. (2006b) \\
\hline LMA525 & 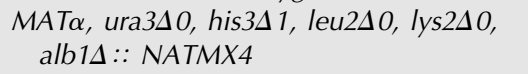 & Lebreton et al. (2006b) \\
\hline LMA401 & 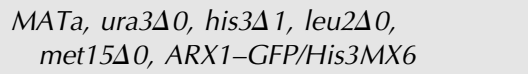 & Lebreton et al. (2006b) \\
\hline LMA411 & 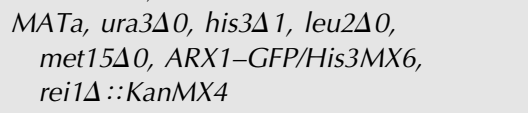 & Lebreton et al. (2006b) \\
\hline ED49 & 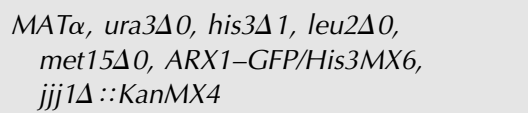 & This study \\
\hline LMA545-B & 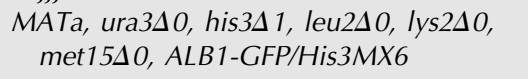 & Lebreton et al. (2006b) \\
\hline LMA545-A & $\begin{array}{l}\text { MATa, ura3 } 30, \text { his } 3 \Delta 1 \text {, leu } 2 \Delta 0, \text { lys } 2 ?, \\
\text { met15?, ALB1-GFP/His3MX6, } \\
\text { rei1 } 1: \text { KanMX4 }\end{array}$ & Lebreton et al. (2006b) \\
\hline ED66 & $\begin{array}{l}\text { MATa, ura3 } \Delta 0, \text { his } 3 \Delta 1, \text { leu2 } 20, \text { lys2?, } \\
\text { met15?, ALB1-GFP/His3MX6, } \\
\text { jjj1 } 1 \Delta: \text { KanMX4 }\end{array}$ & This study \\
\hline OT52 & 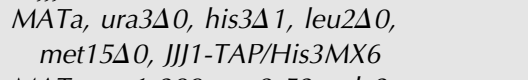 & Winzeler et al. (1999) \\
\hline ED19 & $\begin{array}{l}\text { MATa, trp1-289, ura3-52, ade2, } \\
\text { leu2,-3-112, arg4, JJJ1-TAP/kITRP1, } \\
\text { REI1-13Мyc/KanMX6 }\end{array}$ & This study \\
\hline ED46 & $\begin{array}{l}\text { MATa, trp1-289, ura3-52, ade2, } \\
\text { leu2,-3-112, arg4, REI1-TAP/URA3, } \\
\text { JJJ1-13Myc/kITRP1 }\end{array}$ & This study \\
\hline ED87 & $\begin{array}{l}\text { MATa, trp1-289, ura3-52, ade2, } \\
\text { leu2,-3-112, arg4, RLP24-TAP/ kITRP1, } \\
\text { JJJ1-13Мус/KanMX6 }\end{array}$ & This study \\
\hline LMA352 & $\begin{array}{l}\text { MATa, trp1-289, ura3-52, ade2, } \\
\text { leu2,-3-112, arg4, RLP24-TAP/kITRP1, } \\
\text { REI1-13Myc/KanMX6 }\end{array}$ & Lebreton et al. (2006b) \\
\hline ED82 & $\begin{array}{l}\text { MATa, trp1-289, ura3-52, ade2, } \\
\text { leu2,-3-112, arg4, JJJ1-13Мус/КапМХ6 }\end{array}$ & This study \\
\hline
\end{tabular}

(continued) were indicated by Lebreton et al. (2006a,b). We constructed an empty vector pED13, which was derived from pFL44L plasmid, by elimination of an insert of the library by Kpn1/Sal1 digestion. The pED12 plasmid was generated by cloning JJJ1 extending from 350 base pairs upstream from the initiation codon to $640 \mathrm{bp}$ downstream of the stop codon at the Not1 site. Generation times were calculated from growth curves in liquid culture at $23^{\circ} \mathrm{C}$ during a period of $32 \mathrm{~h}$.

\section{Sucrose gradient sedimentation and Western blotting}

The strains were grown at $30^{\circ} \mathrm{C}$ in appropriate medium to an $\mathrm{A}_{600}$ of $0.3-0.6$, and the mutant strains were shifted at $23^{\circ} \mathrm{C}$ for $2 \mathrm{~h}$ before cycloheximide treatment. Extracts were prepared in $10 \mathrm{mM}$ Tris- $\mathrm{HCl}(\mathrm{pH}$ 7.4), $30 \mathrm{mM} \mathrm{MgCl}_{2}, 100 \mathrm{mM} \mathrm{NaCl}$, and $50 \mu \mathrm{g} / \mathrm{mL}$ cycloheximide using glass beads vortexing. They were fractioned by ultracentrifugation in $10 \%-50 \%$ sucrose gradients for $3 \mathrm{~h}$ at $39,000 \mathrm{rpm}$ at $4^{\circ} \mathrm{C}$ in an SW41 Ti rotor. The fractions were recovered with an ISCO fractionator, and the absorbance at $254 \mathrm{~nm}$ was measured. The proteins were precipitated with 10\% TCA and separated on polyacrylamide-SDS gels.

Nog1 and Rlp24 (Saveanu et al. 2003), Nog2 (Saveanu et al. 2001), Tif6 (Senger et al. 2001), Rpl1 (a gift of F. Lacroute, Gif sur Yvette, France), and Nmd3 (a gift of A.W. Johnson, Austin, TX, USA) native proteins were detected by indirect immunoblotting, using specific polyclonal rabbit antibodies at a 1:5000 dilution (except at 1:10,000 for Rpl1). Specific polyclonal rabbit antibodies against Arx1 and Rei1 were used at 1:700 and 1:2000, respectively (Lebreton et al. 2006b). TAP-tagged proteins were revealed with a 1:10,000 dilution of peroxidase-antiperoxidase complex (PAP; Sigma). GFP-tagged and $13 \mathrm{Myc}$-tagged proteins were detected using anti-GFP 1:700 (rabbit polyclonal antibody, Santa Cruz) and anti-myc 1:1000 (mouse monoclonal antibody, Santa Cruz), respectively. Secondary antibodies (Goat AntiRabbit- or Goat Anti-Mouse-HRP Conjugate from Bio-Rad) were used at a 1:10,000 dilution. Peroxidase activity of the secondary antibodies was revealed using Millipore chemiluminescence HRP substrate system.

\section{High copy number suppressor genetic screen}

The jjj1s strain (ED11) was transformed with a yeast genomic library cloned into a 
TABLE 1. Continued

\begin{tabular}{|c|c|c|}
\hline Strain & Genotype & Reference \\
\hline LMA375 & 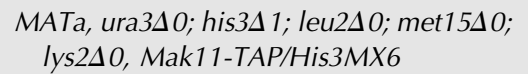 & Saveanu et al. (2007) \\
\hline ED72 & 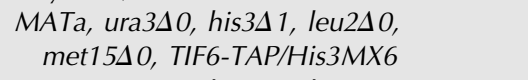 & Winzeler et al. (1999) \\
\hline ED89 & 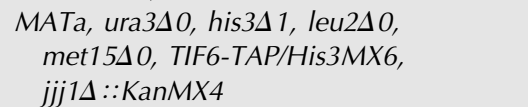 & This study \\
\hline ED92 & 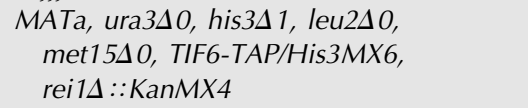 & This study \\
\hline
\end{tabular}

selected on minimal medium without leucine and tryptophan (-LW) plates, and the diploids displaying a positive two-hybrid interaction were selected on minimal medium without leucine, tryptophan, and histine (-LWH) plates. An X-Gal overlay was performed according to FromontRacine et al. (1997) to select the positive two-hybrid clones on the second reporter gene, the LacZ gene.

\section{Tandem affinity purification}

Purifications were performed from $4 \mathrm{~L}$ of yeast culture as described by Rigaut et al. (1999) using classical buffers containing $0.1 \mathrm{M} \mathrm{NaCl}$. The TEV eluates were precipitated with $10 \%$ TCA, and the final TAP purifica-

(URA3) pFL44L plasmid. The transformants were isolated on minimum medium plate without uracil (-URA). The plates were incubated at $30^{\circ} \mathrm{C}$ overnight and then shifted at $20^{\circ} \mathrm{C}$ for several days. The clones that display a slow growth rescue at $20^{\circ} \mathrm{C}$ were selected by comparing the size of the transformants with those transformed with an empty vector. Their plasmidic DNAs were extracted and DNA inserts were sequenced using M13 forward and reverse universal primers. The suppressor plasmids were tested by retransformation of the jijis strain (ED11).

\section{Fluorescence microscopy}

Cells were grown in minimal medium overnight at $30^{\circ} \mathrm{C}$ to an $\mathrm{A}_{600}$ of 0.5 . The mutant strains were shifted to $23^{\circ} \mathrm{C}$ for $2 \mathrm{~h}$. Indirect immunofluorescence of the TAP-tagged fusion proteins was detected using anti-protein A antibodies and $\mathrm{Cy} 3$ secondary antibodies as described (Pringle et al. 1991). Fluorescence was visualized using an epifluorescence microscope (model DMRB; Leica) as described by Lebreton et al. (2006b).

\section{RNA extraction, Northern blotting, and primer extension}

After growth of each yeast strain in rich media at $30^{\circ} \mathrm{C}$ up to an $\mathrm{A}_{600}$ of 0.5 , the cultures were shifted at $23^{\circ} \mathrm{C}$ for 15 min or $2 \mathrm{~h}$. The cultures were centrifuged, and yeast cells were broken using glass beads. RNA extractions were performed with phenol/chloroform. Mature and intermediate large species were separated on $1 \%$ agarose gel and small species were separated on 5\% polyacrylamide-urea gels. RNAs were transferred on Hybond+ membranes, and their identification was performed by hybridization with various ${ }^{32} \mathrm{P}$-labeled oligonucleotides. Primer extensions were performed using ${ }^{32} \mathrm{P}$-labeled oligonucleotides, and the products were separated on $5 \%$ polyacrylamide-urea gels. The sequences of the oligonucleotides were previously described by Saveanu et al. (2001).

\section{Two-hybrid matrix}

The strain CG1945 transformed with the bait Gateway plasmid pAS2 $\Delta \Delta-J J J 1, R E I 1, A R X 1, A L B 1$, and RPL24B was mated with the strain Y187 transformed with the prey Gateway plasmid pActII containing the same open reading frame (ORF). Diploids were tions were precipitated with methanol/chloroform. The purified complexes were separated on a $4 \%-12 \%$ polyacrylamide gradientSDS gel and analyzed by Western blotting as described above.

\section{ACKNOWLEDGMENTS}

We thank Jean-Christophe Rain (Hybrigenics, Paris, France) for performing the yeast two-hybrid screen with Reil as bait, Alice Lebreton (CGM, Gif-sur-Yvette, France) for strains and plasmids, and Caty Gonzalez for technical help. We are grateful to François Lacroute (CGM, Gif-sur-Yvette, France) for providing the highcopy vector genomic S. cerevisiae library, Edouard Bertrand (IGM, Montpellier, France) for providing the two-hybrid Gateway plasmids, and Franco Fasiolo (IBMC, Strasbourg, France), Arlen W. Johnson (MGM, Austin, TX, USA), and François Lacroute for the gift of Tif6, Nmd3, and Rpl1 antibodies, respectively. We thank Helen Neil for critical reading of the manuscript and Cosmin Saveanu for all the scientific discussions and advice for experiments. E.D. and G.L. are grateful to Claire Bonnerot for having introduced them to Jjj1. This work was supported by a grant from the Ministère délégué à l'Enseignement Supérieur et à la Recherche (ACI-BCM0089-2003) to M. F.-R., ARC (33.49) and FRM (FRM-INE20031114111) to G.L. E. D. received a fellowship from La ligue Nationale contre le Cancer.

Received March 27, 2007; accepted June 12, 2007.

\section{REFERENCES}

Albanese, V., Yam, A.Y., Baughman, J., Parnot, C., and Frydman, J. 2006. Systems analyses reveal two chaperone networks with distinct functions in eukaryotic cells. Cell 124: 75-88.

Baudin, A., Ozier-Kalogeropoulos, O., Denouel, A., Lacroute, F., and Cullin, C. 1993. A simple and efficient method for direct gene deletion in Saccharomyces cerevisiae. Nucleic Acids Res. 21: 3329-3330.

Brachmann, C.B., Davies, A., Cost, G.J., Caputo, E., Li, J., Hieter, P., and Boeke, J.D. 1998. Designer deletion strains derived from Saccharomyces cerevisiae S288C: A useful set of strains and plasmids for PCR-mediated gene disruption and other applications. Yeast 14: 115-132.

Decatur, W.A. and Fournier, M.J. 2002. rRNA modifications and ribosome function. Trends Biochem. Sci. 27: 344-351.

Fan, C.Y., Lee, S., and Cyr, D.M. 2003. Mechanisms for regulation of Hsp70 function by Hsp40. Cell Stress Chaperones 8: 309-316. 
Fatica, A. and Tollervey, D. 2002. Making ribosomes. Curr. Opin. Cell Biol. 14: 313-318.

Fotin, A., Cheng, Y., Grigorieff, N., Walz, T., Harrison, S.C., and Kirchhausen, T. 2004. Structure of an auxilin-bound clathrin coat and its implications for the mechanism of uncoating. Nature 432: 649-653.

Fromont-Racine, M., Rain, J.C., and Legrain, P. 1997. Toward a functional analysis of the yeast genome through exhaustive twohybrid screens. Nat. Genet. 16: 277-282.

Fromont-Racine, M., Senger, B., Saveanu, C., and Fasiolo, F. 2003. Ribosome assembly in eukaryotes. Gene 313: 17-42.

Gadal, O., Strau, B.D., Kessl, J., Trumpower, B., Tollervey, D., and Hurt, E. 2001. Nuclear export of 60S ribosomal subunits depends on Xpolp and requires a nuclear export sequence-containing factor, Nmd3p, that associates with the large subunit protein Rpl10p. Mol. Cell. Biol. 21: 3405-3415.

Gasch, A.P., Spellman, P.T., Kao, C.M., Carmel-Harel, O., Eisen, M.B., Storz, G., Botstein, D., and Brown, P.O. 2000. Genomic expression programs in the response of yeast cells to environmental changes. Mol. Biol. Cell 11: 4241-4257.

Gavin, A.C., Aloy, P., Grandi, P., Krause, R., Boesche, M., Marzioch, M., Rau, C., Jensen, L.J., Bastuck, S., Dumpelfeld, B., et al. 2006. Proteome survey reveals modularity of the yeast cell machinery. Nature 440: 631-636.

Hedges, J., West, M., and Johnson, A.W. 2005. Release of the export adapter, Nmd3p, from the $60 \mathrm{~S}$ ribosomal subunit requires Rpl10p and the cytoplasmic GTPase Lsg1p. EMBO J. 24: 567-579.

Hennessy, F., Nicoll, W.S., Zimmermann, R., Cheetham, M.E., and Blatch, G.L. 2005. Not all J domains are created equal: Implications for the specificity of Hsp40-Hsp70 interactions. Protein Sci. 14: 1697-1709.

Ho, J.H., Kallstrom, G., and Johnson, A.W. 2000. Nmd3p is a Crm1pdependent adapter protein for nuclear export of the large ribosomal subunit. J. Cell Biol. 151: 1057-1066.

Huh, W.K., Falvo, J.V., Gerke, L.C., Carroll, A.S., Howson, R.W., Weissman, J.S., and O'Shea, E.K. 2003. Global analysis of protein localization in budding yeast. Nature 425: 686-691.

Hung, N.J. and Johnson, A.W. 2006. Nuclear recycling of the pre-60S ribosomal subunit-associated factor Arxl depends on Reil in Saccharomyces cerevisiae. Mol. Cell. Biol. 26: 3718-3727.

Lebreton, A., Saveanu, C., Decourty, L., Jacquier, A., and FromontRacine, M. 2006a. NSA2 is an unstable, conserved factor required for the maturation of 27 SB pre-rRNAs. J Biol Chem. 281: 2709927108.

Lebreton, A., Saveanu, C., Decourty, L., Rain, J.C., Jacquier, A., and Fromont-Racine, M. 2006b. A functional network involved in the recycling of nucleocytoplasmic pre-60S factors. J. Cell Biol. 173: 349-360.

Leslie, D.M., Zhang, W., Timney, B.L., Chait, B.T., Rout, M.P., Wozniak, R.W., and Aitchison, J.D. 2004. Characterization of karyopherin cargoes reveals unique mechanisms of Kap121pmediated nuclear import. Mol. Cell. Biol. 24: 8487-8503.

Meyer, A.E., Hung, N.J., Yang, P., Johnson, A.W., and Craig, E.A. 2007. The specialized cytosolic J-protein, Jjj1, functions in $60 \mathrm{~S}$ ribosomal subunit biogenesis. Proc. Natl. Acad. Sci. 104: 1558-1563.
Nissan, T.A., Bassler, J., Petfalski, E., Tollervey, D., and Hurt, E. 2002. $60 \mathrm{~S}$ pre-ribosome formation viewed from assembly in the nucleolus until export to the cytoplasm. EMBO J. 21: 55395547.

Pishvaee, B., Costaguta, G., Yeung, B.G., Ryazantsev, S., Greener, T., Greene, L.E., Eisenberg, E., McCaffery, J.M., and Payne, G.S. 2000. A yeast DNA J protein required for uncoating of clathrin-coated vesicles in vivo. Nat. Cell Biol. 2: 958-963.

Pringle, J.R., Adams, A.E., Drubin, D.G., and Haarer, B.K. 1991. Immunofluorescence methods for yeast. Methods Enzymol. 194: 565-602.

Rigaut, G., Shevchenko, A., Rutz, B., Wilm, M., Mann, M., and Seraphin, B. 1999. A generic protein purification method for protein complex characterization and proteome exploration. Nat. Biotechnol. 17: 1030-1032.

Rout, M.P., Blobel, G., Aitchison, J.D., and Tyers, M. 1997. A distinct nuclear import pathway used by ribosomal proteins. Cell 89: $715-725$.

Saveanu, C., Bienvenu, D., Namane, A., Gleizes, P.E., Gas, N., Jacquier, A., and Fromont-Racine, M. 2001. Nog2p, a putative GTPase associated with pre-60S subunits and required for late 60S maturation steps. EMBO J. 20: 6475-6484.

Saveanu, C., Namane, A., Gleizes, P.E., Lebreton, A., Rousselle, J.C., Noaillac-Depeyre, J., Gas, N., Jacquier, A., and FromontRacine, M. 2003. Sequential protein association with nascent $60 S$ ribosomal particles. Mol. Cell. Biol. 23: 4449-4460.

Saveanu, C., Rousselle, J.C., Lenormand, P., Namane, A., Jacquier, A., and Fromont-Racine, M. 2007. The p21-activated protein kinase inhibitor Skb15 and its budding yeast homologue are $60 \mathrm{~S}$ ribosome assembly factors. Mol. Cell. Biol. 27: 2897-2909.

Senger, B., Lafontaine, D.L., Graindorge, J.S., Gadal, O., Camasses, A., Sanni, A., Garnier, J.M., Breitenbach, M., Hurt, E., and Fasiolo, F. 2001. The nucle(ol)ar Tif6p and Efllp are required for a late cytoplasmic step of ribosome synthesis. Mol. Cell 8: 1363-1373.

Tschochner, H. and Hurt, E. 2003. Pre-ribosomes on the road from the nucleolus to the cytoplasm. Trends Cell Biol. 13: 255-263.

Ungewickell, E., Ungewickell, H., Holstein, S.E., Lindner, R., Prasad, K., Barouch, W., Martin, B., Greene, L.E., and Eisenberg, E. 1995. Role of auxilin in uncoating clathrin-coated vesicles. Nature 378: 632-635.

Venema, J. and Tollervey, D. 1999. Ribosome synthesis in Saccharomyces cerevisiae. Annu. Rev. Genet. 33: 261-311.

Warner, J.R. 1999. The economics of ribosome biosynthesis in yeast. Trends Biochem. Sci. 24: 437-440.

Winzeler, E.A., Shoemaker, D.D., Astromoff, A., Liang, H., Anderson, K., Andre, B., Bangham, R., Benito, R., Boeke, J.D., Bussey, H., et al. 1999. Functional characterization of the $S$. cerevisiae genome by gene deletion and parallel analysis. Science 285: 901-906.

Zakalskiy, A., Hogenauer, G., Ishikawa, T., Wehrschutz-Sigl, E., Wendler, F., Teis, D., Zisser, G., Steven, A.C., and Bergler, H. 2002. Structural and enzymatic properties of the AAA protein Drglp from Saccharomyces cerevisiae. Decoupling of intracellular function from ATPase activity and hexamerization. J. Biol. Chem. 277: 26788-26795. 

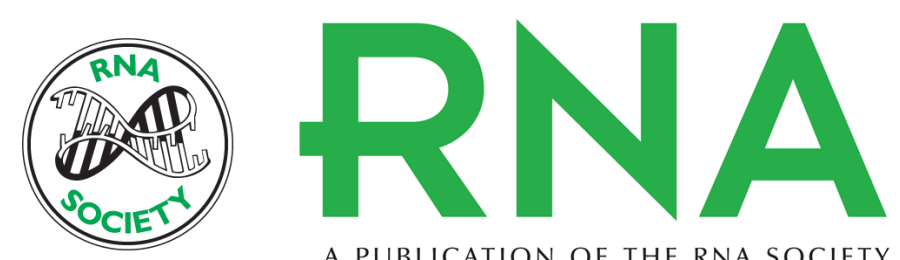

A PUBLICATION OF THE RNA SOCIETY

\section{The Hsp40 chaperone Jjj1 is required for the nucleo-cytoplasmic recycling of preribosomal factors in Saccharomyces cerevisiae}

Emilie Demoinet, Alain Jacquier, Georges Lutfalla, et al.

RNA 2007 13: 1570-1581 originally published online July 24, 2007

Access the most recent version at doi:10.1261/rna.585007

References This article cites 36 articles, 15 of which can be accessed free at:

http://rnajournal.cshlp.org/content/13/9/1570.full.html\#ref-list-1

License

Email Alerting Receive free email alerts when new articles cite this article - sign up in the box at the Service top right corner of the article or click here.

To subscribe to $R N A$ go to:

http://rnajournal.cshlp.org/subscriptions 